Claremont Colleges

Scholarship@ Claremont

All HMC Faculty Publications and Research

HMC Faculty Scholarship

$5-1-2008$

\title{
An Alternate Approach to Alternating Sums: A Method to DIE for
}

Arthur T. Benjamin

Harvey Mudd College

Jennifer J. Quinn

University of Washington - Tacoma Campus

\section{Recommended Citation}

Benjamin, Arthur T. and Jennifer J. Quinn. "An Alternate Approach to Alternating Sums: A Method to Die For" The College Mathematics Journal, Vol. 39, No. 3. pp. 191-201, September 2008.

This Article is brought to you for free and open access by the HMC Faculty Scholarship at Scholarship @ Claremont. It has been accepted for inclusion in All HMC Faculty Publications and Research by an authorized administrator of Scholarship @ Claremont. For more information, please contact scholarship@cuc.claremont.edu. 


\section{MAA}

An Alternate Approach to Alternating Sums: A Method to DIE for

Author(s): Arthur T. Benjamin and Jennifer J. Quinn

Source: The College Mathematics Journal, Vol. 39, No. 3 (May, 2008), pp. 191-202

Published by: Mathematical Association of America

Stable URL: http://www.jstor.org/stable/27646625

Accessed: 14/06/2013 16:56

Your use of the JSTOR archive indicates your acceptance of the Terms \& Conditions of Use, available at

http://www.jstor.org/page/info/about/policies/terms.jsp

JSTOR is a not-for-profit service that helps scholars, researchers, and students discover, use, and build upon a wide range of content in a trusted digital archive. We use information technology and tools to increase productivity and facilitate new forms of scholarship. For more information about JSTOR, please contact support@jstor.org. 


\section{An Alternate Approach to Alternating Sums: A Method to DIE for}

Arthur T. Benjamin and Jennifer J. Quinn
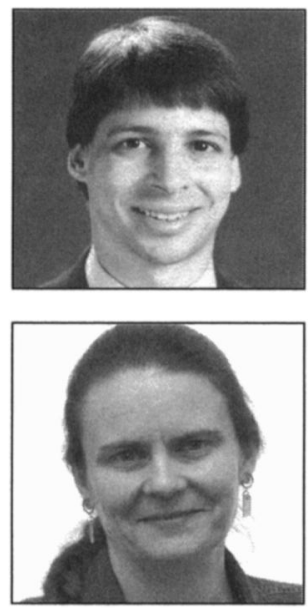

Arthur Benjamin (benjamin @ hmc.edu) received his $\mathrm{PhD}$ from Johns Hopkins University in 1989. He is a Professor of Mathematics at Harvey Mudd College, where he has taught since 1989.

Jennifer Quinn (jjquinn@u.washington.edu) received her $\mathrm{PhD}$ from the University of Wisconsin in 1993. She is a Professor of Interdisciplinary Arts \& Sciences at the University of Washington, Tacoma, where she is building a mathematics curriculum on the expanding campus.

Art and Jenny have written more than 20 research articles together, primarily on combinatorics. In 2006, their book Proofs That Really Count: The Art of Combinatorial Proof received the Beckenbach book prize from the MAA. Currently, they are co-Editors of Math Horizons magazine. They are both recipients of the MAA's Haimo Award for Distinguished College or University Teaching.

\section{Obsession and confession}

Some people think we are fanatics about combinatorial proofs. Ok, we admit it, we are. When we see an identity with a finite sum and an attractive closed form, we are not satisfied until we understand what the identity is counting. For example, the identity obtained by summing the $n$th row of Pascal's triangle,

$$
\sum_{k=0}^{n}\left(\begin{array}{l}
n \\
k
\end{array}\right)=2^{n}
$$

counts the number of subsets of $\{1,2, \ldots, n\}$ in two different ways. (The left side counts the number of subsets of each size $k$ from 0 to $n$. The right side counts subsets by deciding, for each element, whether or not it is in the subset.)

But what if the sum has negative entries? For instance, if you saw the sum

$$
\sum_{k=0}^{m}\left(\begin{array}{l}
n \\
k
\end{array}\right)(-1)^{k}=(-1)^{m}\left(\begin{array}{c}
n-1 \\
m
\end{array}\right),
$$

or a scarier looking sum (from a recent problem in this journal [5]) like

$$
\sum_{j=1}^{n} \sum_{k=1}^{n}(-1)^{j+k}\left(\begin{array}{c}
n-1 \\
j-1
\end{array}\right)\left(\begin{array}{c}
n-1 \\
k-1
\end{array}\right)\left(\begin{array}{c}
j+k \\
j
\end{array}\right)=2,
$$

would you be inclined to try to prove these by counting arguments? Probably not. 
We confess that there was a time that when we saw alternating sum identities like the ones above, we would attack them with noncombinatorial proof techniques, like induction or generating functions. After all, how can an object be added a negative number of times? Perhaps it can be tackled using the Principle of Inclusion-Exclusion (abbreviated P.I.E.), but that brings another level of complexity to the combinatorial proof. But now we have an alternate opinion. Now when we see an alternating sum, we can usually prove it combinatorially with a method that is as easy as pie and even easier than P.I.E.! As we'll demonstrate, this technique offers new insights to identities involving binomial coefficients, Fibonacci numbers, derangements, and other combinatorial structures.

\section{Solution by involution}

We begin with an easy, but important, example. Consider the alternating sum of the $n$th row of Pascal's triangle,

$$
\sum_{k=0}^{n}\left(\begin{array}{l}
n \\
k
\end{array}\right)(-1)^{k}=0
$$

Combinatorially, this identity says that the number of even-sized subsets of $\{1,2, \ldots, n\}$ minus the number of odd-sized subsets of $\{1,2, \ldots, n\}$ is zero, that is, there are as many even subsets as odd subsets. For example, when $n=4$, we list the even subsets on the left and the odd subsets on the right.

\begin{tabular}{cc} 
even & odd \\
\hline$\varnothing$ & 1 \\
12 & 2 \\
13 & 3 \\
14 & 4 \\
23 & 123 \\
24 & 124 \\
34 & 134 \\
1234 & 234
\end{tabular}

Can you find a rule that matches each subset $X$ with its corresponding subset on the other side? Sure. Simply toggle the number 1: If a 1 is in $X$, take it out; if it's not in $X$, put it in. We call this corresponding subset $X \oplus 1$ (a shorthand for the symmetric difference of sets $X \oplus\{1\}$ ). Note that the sizes of $X$ and $X \oplus 1$ differ by \pm 1 . In general, since every subset $X$ in $\{1,2, \ldots, n\}$ "holds hands" with a subset of opposite parity, namely $X \oplus 1$, this shows that there are equal numbers of subsets of even and odd parity. So the identity holds.

The toggle function $f(x)=X \oplus 1$ is an example of a sign-reversing involution. An involution is a function $f$ with the property that $f(f(x))=x$, for all inputs $x$. An involution $f$ is sign-reversing when $x$ and $f(x)$ are always given opposite signs in the alternating sum. The toggle function is an involution since $(X \oplus 1) \oplus 1=X$ for all subsets $X$, and it is sign-reversing since $X$ and $X \oplus 1$ have opposite parity. In contrast, the involution that maps $X$ to its complement in $\{1,2, \ldots, n\}$ is not sign-reversing when $n$ is even. 
Now for $m, n \geq 0$, consider the partial sum $\sum_{k=0}^{m}\left(\begin{array}{l}n \\ k\end{array}\right)(-1)^{k}$. Here, we are looking at all subsets of $\{1,2, \ldots, n\}$ that have at most $m$ elements. Again we will try to pair up the even subsets with the odd ones using the function $f(X)=X \oplus 1$. This works fine except when $f(X)$ has more than $m$ elements (and therefore $f(X)$ is undefined). These exceptions occur whenever $X$ has exactly $m$ elements and $X$ does not contain the element 1 . The number of ways this can happen is $\left(\begin{array}{c}n-1 \\ m\end{array}\right)$, and each of these subsets has the same sign in the summation, namely $(-1)^{m}$, since each exception has $m$ elements. It follows that

$$
\sum_{k=0}^{m}\left(\begin{array}{l}
n \\
k
\end{array}\right)(-1)^{k}=(-1)^{m}\left(\begin{array}{c}
n-1 \\
m
\end{array}\right),
$$

as desired.

\section{The D.I.E. method}

Combinatorialist extraordinaire Doron Zeilberger sometimes refers to our last solution as a "killing involution," since every object $X$ is annihilated in the summation by an object of opposite sign $f(X)$, except for the survivors who did not participate in the involution revolution. Although we prefer the more peaceful hand-holding interpretation, the acronym for our method is just as violent: D.I.E., which stands for Description, Involution, Exception.

Description: Describe a set of objects that is being counted by the sum when we ignore the sign.

Involution: Find an involution between the objects that are counted positively and the objects that are counted negatively in the sum.

Exception: Describe the exceptions, where the involution is undefined. Count these exceptions and note their sign.

For example, let's use the D.I.E. method to prove the following generalization of identity (1). For $0 \leq m<n$,

$$
\sum_{k=0}^{n}\left(\begin{array}{l}
n \\
k
\end{array}\right)\left(\begin{array}{l}
k \\
m
\end{array}\right)(-1)^{k}=0 .
$$

Ignoring the sign factor, we see that for a fixed choice of $k$ between 0 and $n$, $\left(\begin{array}{l}n \\ k\end{array}\right)\left(\begin{array}{l}k \\ m\end{array}\right)$ answers the question "From a class of $n$ students, in how many ways can you choose a committee $X$ of size $k$ and then choose a committee $Y$ of size $m$ that is a subcommittee of $X$ ?" Since the right side of the identity is zero, the challenge (the fun part) is to find a simple way to pair up each $(X, Y)$ object with another object $\left(X^{\prime}, Y^{\prime}\right.$ ) (where $Y^{\prime}$ is a size- $m$ subset of $X^{\prime}$ ) so that the sizes of $X$ and $X^{\prime}$ are of opposite parity. Can we toggle the number 1 , like before, and pair up $(X, Y)$ with $(X \oplus 1, Y)$ ? Not quite. Consider what happens when we try with $n=10, k=5$, and $m=3$. If $(X, Y)=(\{1,2,3,4,5\},\{2,3,5\})$, then toggling with 1 gives $(X \oplus 1, Y)=$ $(\{2,3,4,5\},\{2,3,5\}) . Y$ is a subset of $X \oplus 1$ and the parity of the first subset changed from odd to even. So far so good. If $(X, Y)=(\{6,7,8,9,10\},\{6,8,10\})$, then toggling with 1 gives $(X \oplus 1, Y)=(\{1,6,7,8,9,10\},\{6,8,10\})$. Again, this poses no problems-everything works fine, provided 1 is missing from $Y$. But if $(X, Y)=$ $(\{1,3,5,7,9\},\{1,5,9\})$, then $Y$ is not a subset of $X \oplus 1$ and our hope for a bijective involution is squashed. In general, when 1 is an element of $Y$ (and therefore also 
an element of $X), Y$ is never a subset of $X \oplus 1$. So picking a fixed number like 1 to toggle with $X$ does not create the desired pairing. However, since $m$ is strictly less than $n$, there is at least one number missing from $Y$. This forms the basis of the following solution.

Description: For a given value of $k$ between 0 and $n,\left(\begin{array}{l}n \\ k\end{array}\right)\left(\begin{array}{l}k \\ m\end{array}\right)$ counts ordered pairs $(X, Y)$ such that $Y \subseteq X \subseteq\{1,2, \ldots, n\},|X|=k$, and $|Y|=m$. So $\sum_{k=0}^{n}\left(\begin{array}{l}n \\ k\end{array}\right)\left(\begin{array}{l}k \\ m\end{array}\right)$ counts all ordered pairs $(X, Y)$ where $Y \subseteq X$, the size of $Y$ is $m$, and the size of $X$ ranges from zero to $n$.

Involution: Given $(X, Y)$, let $x$ be the smallest element of $\{1,2, \ldots, n\}$ such that $x$ is not in $Y$. Then define the involution $f(X, Y)=(X \oplus x, Y)$. Note that since $x$ is not in $Y, Y$ remains a subset of $X \oplus x$.

Exception: Since $m<n$, the element $x$ always exists, so the involution above is always defined. Thus there are no exceptions, the involution is a bijection, and the right side of the identity is zero.

The D.I.E. method has a venerable history. It was first used by Fabian Franklin (in [3]) to provide an elegant proof of Euler's pentagonal number theorem. (See [6] for a nice expostion.) Franklin was one of the first graduate students trained in mathematics in the United States. As described in [7], his proof caught the attention of leading European mathematicians, and demonstrated to them that mathematicians trained in America were capable of contributing to research-level mathematics.

\section{Fibonacci, binomials, and polynomials}

Fibonacci numbers are ideally suited for combinatorial arguments. Defining $f_{n}=$ $f_{n-1}+f_{n-2}$ with initial conditions, $f_{0}=f_{1}=1$, it is easy to prove (as in [1], [2]) that $f_{n}$ counts the ways to tile a $1 \times n$ strip of length $n$ with squares (of length 1 ) and dominoes (of length 2). For example, $f_{4}=5$ counts the tilings given in Figure 1.

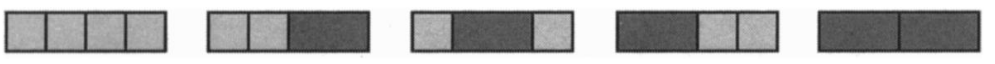

Figure 1. The Fibonacci number $f_{4}=5$ counts the 5 tilings of length 4 .

It has long been known that the partial sums of the sequence of Fibonacci numbers has a beautiful closed form (specifically $\sum_{k=0}^{n} f_{k}=f_{n+2}-1$ ). Is the same true for the alternating sequence of Fibonacci numbers? Absolutely, and D.I.E. will show us the way. For $n \geq 1$, we show that

$$
\sum_{k=1}^{n}(-1)^{k} f_{k}=(-1)^{n} f_{n-1}
$$

Description: Since $f_{k}$ counts tilings of length $k, \sum_{k=1}^{n} f_{k}$ counts tilings of all positive lengths up to length $n$.

Involution: What is the second easiest way to change the parity of the length of a tiling $T$, where $T$ has length at most $n$ ? The easiest way would be to append a square, but that would not be an involution. Instead toggle the. last tile of $T$ : If $T$ ends in a square, turn that square into a domino; if $T$ ends in a domino, turn that domino into a square. This is clearly an involution. 
Exception: The only exceptions occur when $T$ has maximum length $n$ and ends in a square. In this case, toggling the last tile produces a tiling of length $n+1$, which is too long for our consideration. The number of tilings of length $n$ that end with a square is $f_{n-1}$, and all of these tilings are assigned a sign of $(-1)^{n}$ in our summation (since they have length $n$ ). Hence, the right side of the identity is $(-1)^{n} f_{n-1}$, as desired.

Delightful identities frequently occur when the involution is not a bijection. But keeping track of the exceptions can be a challenge. To further illustrate, we consider the previous alternating sum with the new twist of multiplying each Fibonacci number by its index. How can D.I.E. bring

$$
\sum_{k=1}^{n}(-1)^{k} k f_{k}
$$

into the light?

Description: For a given value of $k$ between 1 and $n, k f_{k}$ counts tilings of length $k$ where a coin is placed somewhere on top of the tiling, so there are $k$ choices for the location of the coin. So $\sum_{k=1}^{n} k f_{k}$ counts tilings of any positive length up to length $n$ with a coin on top.

Involution: Given a tiling $T$ of length at most $n$, toggle the last tile as before (replace a final square with a domino or a final domino with a square) while preserving the position of the coin.

Exception: Two types of exceptions occur: either $T$ has the maximum possible length $n$ and ends in a square or $T$ has length $k$ and ends in a domino with a coin above cell $k$. (In the latter case, toggling the last tile would result in the coin being suspended in mid-air.) There are $n f_{n-1}$ exceptions of the first type (since the coin can go on any of the $n$ cells). For exceptions of the second type we have, for $2 \leq j \leq n, f_{j-2}$ tilings of length $j$ that end with a domino, each with a sign of $(-1)^{j}$. This gives $f_{0}-f_{1}+f_{2}-$ $\cdots+(-1)^{n} f_{n-2}$ exceptions of the second type. By the previous identity this reduces to $1+(-1)^{n-2} f_{n-3}$. Combining the number of exceptions of both types gives this identity: For $n \geq 3$,

$$
\sum_{k=1}^{n}(-1)^{k} k f_{k}=(-1)^{n}\left(n f_{n-1}+f_{n-3}\right)+1 .
$$

Fibonacci numbers interact with binomial coefficients in many beautiful ways. Perhaps the simplest of these comes from the sum of the diagonals of Pascal's Triangle (see Figure 2).

The pattern is unmistakable:

$$
\sum_{k \geq 0}\left(\begin{array}{c}
n-k \\
k
\end{array}\right)=f_{n}
$$

A quick combinatorial proof of this is based on the fact that the number of length- $n$ tilings using exactly $k$ dominoes is $\left(\begin{array}{c}n-k \\ k\end{array}\right)$. To see why, note that such a tiling contains $k$ dominoes and $n-2 k$ squares, and thus uses a total of $n-k$ tiles. From these $n-k$ tiles, we choose $k$ of them to be dominoes, which can be done in $\left(\begin{array}{c}n-k \\ k\end{array}\right)$ ways. (We note that when $k$ exceeds $n / 2,\left(\begin{array}{c}n-k \\ k\end{array}\right)=0$, which makes sense combinatorially, since the number of dominoes is at most $n / 2$.)

Now consider the alternating sum $\sum_{k \geq 0}(-1)^{k}\left(\begin{array}{c}n-k \\ k\end{array}\right)$. 


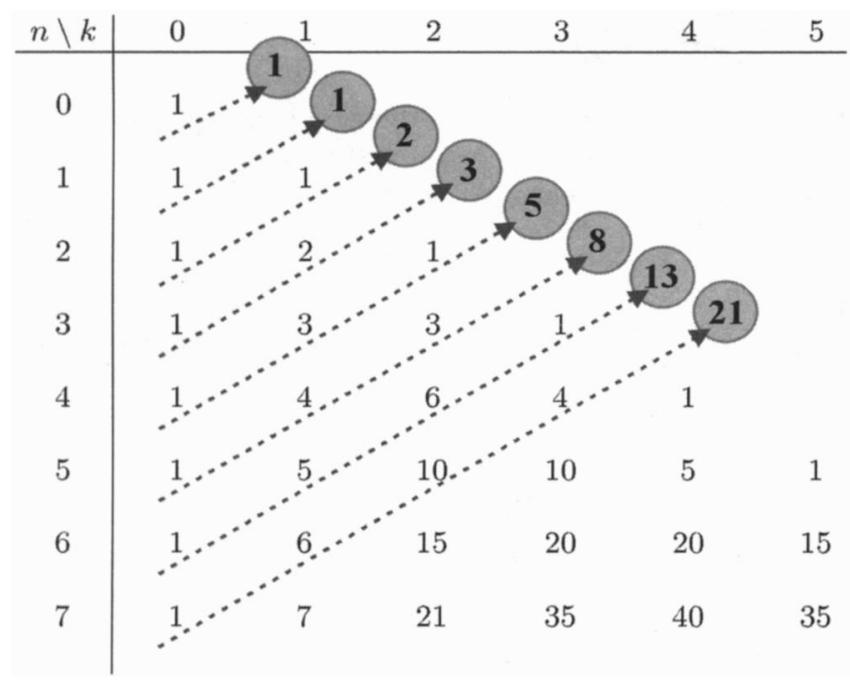

Figure 2. Diagonal sums of Pascal's Triangle.

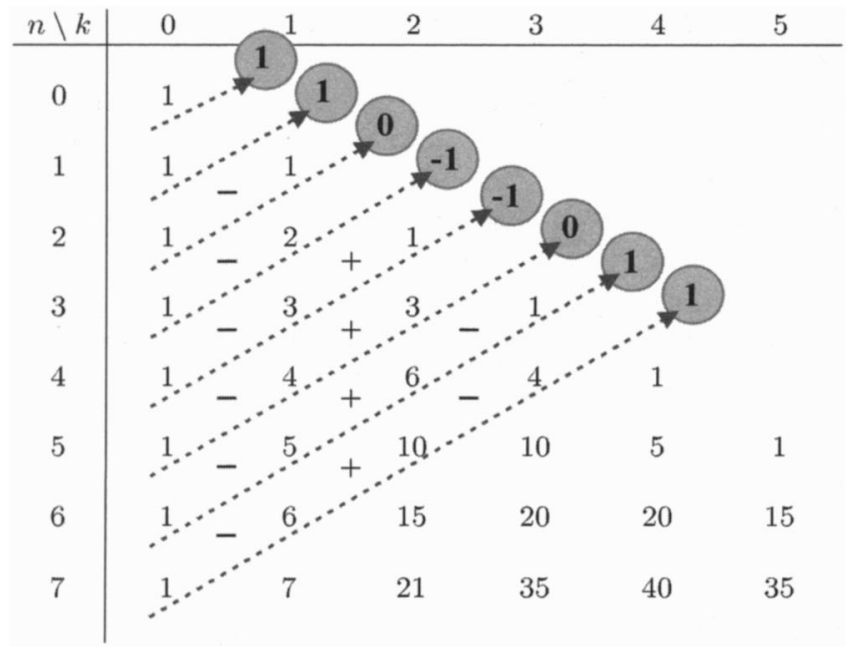

Figure 3. Diagonal alternating sums of Pascal's Triangle.

Here the data (see Figure 3) suggest that the right-hand side is always 0,1 , or -1 . Combinatorially, this suggests that the number of length- $n$ tilings containing an even number of dominoes is about the same as the number containing an odd number of dominoes. Given a length $n$ tiling $T$, how can we change the parity of the number of dominoes without changing the length of $T$ ? If $T$ begins with a domino, we can replace the initial domino with two squares. Likewise, if $T$ begins with two squares, we can replace them with a domino, as illustrated in Figure 4.

But what if $T$ begins with $s d$, a square followed by a domino? Then we ignore these two tiles and look at what happens next. That is, if the tiling begins $s d d$, we replace that string with $s d s s$, and vice versa. Now we are covered unless the tiling begins $s d s d$ 


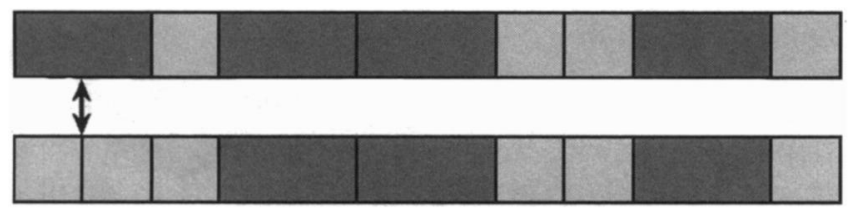

Figure 4. We can toggle an initial domino with an initial pair of squares.

(abbreviated $\left.(s d)^{2}\right)$, in which case we look at what happens after that. Continuing in this way, we arrive at this identity: For $n \geq 0$,

$$
\sum_{k \geq 0}(-1)^{k}\left(\begin{array}{c}
n-k \\
k
\end{array}\right)=\left\{\begin{array}{rll}
0 & \text { if } n \equiv 2 \text { or } 5 & (\bmod 6) \\
1 & \text { if } n \equiv 0 \text { or } 1 & (\bmod 6) \\
-1 & \text { if } n \equiv 3 \text { or } 4 & (\bmod 6)
\end{array}\right.
$$

Description: For a given value of $k,\left(\begin{array}{c}n-k \\ k\end{array}\right)$ counts square-domino tilings of length $n$ with exactly $k$ dominoes. So $\sum_{k>0}\left(\begin{array}{c}n-k \\ k\end{array}\right)$ counts all tilings of length $n$.

Involution: Let $T$ be a tiling of length $n$. If $T$ begins with exactly $j \geq 0$ squaredomino pairs, then we write $T=(s d)^{j} U$, where $U$ is a tiling of length $n-3 j$ that does not begin with $s d$. If $U$ begins with a domino, then replace that domino with two squares; if $U$ begins with two squares, then replace these two squares with a domino. This involution changes the number of dominoes by \pm 1 .

Exception: There is at most one exception, which occurs when $U$ is empty or contains a single square, depending on the value of $n(\bmod 3)$. Here $T=(s d)^{\lfloor n / 3\rfloor}$ or $T=$ $(s d)^{\lfloor n / 3\rfloor} s$. Notice that if $n \equiv 2(\bmod 3)($ that is, $n \equiv 2$ or $5(\bmod 6))$, then no exceptions are possible. If $n \equiv 0$ or $1(\bmod 6)$, say $n=6 t$ or $6 t+1$, then the sole exception, either $T=(s d)^{2 t}$ or $(s d)^{2 t} s$, contains $2 t$ dominoes. This exceptional tiling contains an even number of dominoes and as such contributes +1 to the alternating sum. If $n \equiv 3$ or $4(\bmod 6)$, say $n=6 t+3$ or $6 t+4$, then the sole exception, $T=(s d)^{2 t+1}$ or $(s d)^{2 t+1} s$, contains $2 t+1$ dominoes. Here the exceptional tiling contains an odd number of dominoes and contributes -1 to the alternating sum.

Now consider the similar-looking polynomial identity

$$
\sum_{k \geq 0}(-1)^{k}\left(\begin{array}{c}
n-k \\
k
\end{array}\right)(x y)^{k}(x+y)^{n-2 k}=\sum_{j \geq 0} x^{n-j} y^{j},
$$

presented in [9] by algebraic arguments. Since both sides of (2) are polynomials, verifying the equality for all nonnegative integer values of $x$ and $y$ is sufficient to prove the identity. We will modify the previous D.I.E. argument-giving combinatorial meaning to the indeterminants $x$ and $y$.

Description: Given nonnegative integers $x, y$, and $k,\left(\begin{array}{c}n-k \\ k\end{array}\right)(x y)^{k}(x+y)^{n-2 k}$ counts square-domino tilings of length $n$ with $k$ dominoes (and $n-2 k$ squares) in which each tile is painted with one of $x$ light colors or one of $y$ dark colors as follows: Squares can be painted with any of the $x+y$ colors. The left half of a domino is painted one of $x$ light colors and the right half is painted one of $y$ dark colors, so a domino can be painted $x y$ ways. The sum $\sum_{k \geq 0}\left(\begin{array}{c}n-k \\ k\end{array}\right)(x y)^{k}(x+y)^{n-2 k}$ counts all square-domino tilings of length $n$ subject to the tile painting restrictions using $x$ light and $y$ dark colors. 
Involution: Scan the tiling from left to right until you encounter a domino or a light square followed immediately by a dark square. If a domino occurs first, transform it to a light square-dark square combination by chopping the domino down the middle; if a light-dark pair of squares appears first, transform them to a domino by gluing them together. This process is clearly reversible and changes the parity of the number of dominoes in the tiling.

Exception: The exceptions are those tilings with no dominoes consisting of $j$ dark squares followed by $n-j$ light squares, for some $j \geq 0$. There are $\sum_{j \geq 0} x^{n-j} y^{j}$ such tilings, all of which are counted positively since they contain no dominoes.

\section{P.I.E. can D.I.E.!}

Alternating sums arise in combinatorial problems solved using the Principle of Inclusion-Exclusion (P.I.E.) as described in every combinatorics textbook. For instance, the number of onto functions from an $m$-element set to an $n$-element set is given by

$$
\begin{aligned}
f(m, n) & =n^{m}-n(n-1)^{m}+\left(\begin{array}{l}
n \\
2
\end{array}\right)(n-2)^{m}-\left(\begin{array}{l}
n \\
3
\end{array}\right)(n-3)^{m}+\cdots \\
& =\sum_{k=0}^{n}(-1)^{k}\left(\begin{array}{l}
n \\
k
\end{array}\right)(n-k)^{m},
\end{aligned}
$$

where the first term in the sum counts all functions from $\{1,2, \ldots, m\}$ to $\{1,2, \ldots, n\}$ with no restrictions. From this, we subtract those functions that do not hit element 1 (there are $(n-1)^{m}$ of these), as well as those functions that miss 2 , those that miss 3 , up to those that miss $n$. But we need to add back those functions that miss 1 and 2 (there are $(n-2)^{m}$ of these), and so on.

But this can also be proved using D.I.E. just as easily.

Description: Given $k, m, n \geq 0,\left(\begin{array}{l}n \\ k\end{array}\right)(n-k)^{m}$ counts the ways to select a $k$-element subset $X \subseteq\{1,2, \ldots, n\}$ and then create an $m$-letter word $Y$ from the elements of $\{1,2, \ldots, n\}-X$. For example, if $n=9, m=6$, and $k=2$, a typical object is $(X, Y)=(\{2,8\}, 314159)$. The unsigned summ, $\sum_{k=0}^{n}\left(\begin{array}{l}n \\ k\end{array}\right)(n-k)^{m}$, counts all pairs $(X, Y)$ where $X$ is any subset of $\{1,2, \ldots, n\}$ and $Y$ is an $m$-letter word created from elements in the complement of $X$.

Involution: Given $(X, Y)$ let $x$ be the smallest number in $\{1,2, \ldots, n\}$ that does not appear in $Y$. Match $(X, Y)$ to $(X \oplus x, Y) . Y$ is also an $m$-letter word made from the elements of $\{1,2, \ldots, n\}-(X \oplus x)$ and $x$ is still the smallest element of $\{1,2, \ldots, n\}$ missing from $Y$. Furthermore, the size of $X \oplus x$ differs from the size of $X$ by \pm 1 . (Our example $(\{2,8\}, 314159)$ has $x=2$ and $k=2$, and it will be matched to $(\{8\}, 314159)$, which has $x=2$ and $k=1$.)

Exception: The involution fails when no elements of $\{1,2, \ldots, n\}$ are missing from $Y$. This requires $X$ to be the empty set and $Y$ to contain every letter from $\{1,2, \ldots, n\}$. There are $f(m, n)$ such exceptions, and since $k=0$ for these, each of them is counted positively.

Another classical application of P.I.E. is counting the derangements of $\{1,2, \ldots, n\}$, the number of ways to arrange the integers 1 through $n$ so that no integer lies in its natural position, that is, 1 is not first, 2 is not second, and so on. We let $D_{n}$ denote the number of derangements of $\{1,2, \ldots, n\}$. As is well known to all P.I.E.-lovers, 


$$
D_{n}=\sum_{k=0}^{n}(-1)^{k} \frac{n !}{k !} .
$$

Here we present a simple proof using D.I.E.

Description: Given nonnegative integers $k \leq n, \frac{n !}{k !}$ counts $(n-k)$-letter words created from elements of $\{1,2, \ldots, n\}$ where no letter is repeated. For $n=9$, a typical word might be $X=497$ (when $k=6$ ) or $X=314592687$ (when $k=0$ ). The unsigned sum $\sum_{k=0}^{n} \frac{n !}{k !}$ counts all words created from $\{1,2, \ldots, n\}$ without repetition (including the empty word).

Involution: Given a word $X$ of length $n-k$, we pair it with a new word $X^{\prime}$ as follows. Let $\zeta(X)$ be the smallest number that is either absent from $X$ or in its natural position. If $\zeta(X)$ is absent from $X$, then insert $\zeta(X)$ in its natural position. If $\zeta(X)$ is in its natural position, then remove it from $X$. The lengths of $X$ and $X^{\prime}$ differ by one and $\zeta(X)=$ $\zeta\left(X^{\prime}\right)$ since neither action can result in a number smaller than $\zeta(X)$ moving into its natural position. For example, $\zeta(497)=1$, so 497 is paired with $1497 ; \zeta(3145)=2$, so 3145 is paired with 32145 ; and $\zeta(314592687)=8$, so 314592687 is paired with 31459267.

Exception: The only exceptions occur for those words $X$ where $\zeta(X)$ does not exist. These words have no missing numbers (so $X$ has length $n$ ) and no number is in its natural position. Hence there are $D_{n}$ exceptions, and since each has length $n$ (so $k=0$ ), they are counted positively in the summation.

Indeed, the Principle of Inclusion-Exclusion itself can be proved using D.I.E. Given a set of objects $S$, and a set of (undesirable) properties $P_{1}, P_{2}, \ldots, P_{n}$ that some of the objects in $S$ possess, P.I.E. says that the number of objects in $S$ with no undesirable properties is

$$
\sum_{T \subseteq\{1,2, \ldots, n\}}(-1)^{|T|} f(T)
$$

where $f(T)$ is the number of objects that have all of the properties $P_{j}$ for $j$ in $T$, and possibly other properties as well. For example, in the derangements problem, $S$ is the set of all permutations of $\{1,2, \ldots, n\}$ and a permutation has property $P_{j}$ if the number $j$ is in its natural position. Here $f(\{2,3,5,8\})=(n-4)$ ! counts those permutations where the numbers $2,3,5$, and 8 (and possibly others too) are in their natural positions.

We now prove P.I.E. by D.I.E.

Description: Given a subset $T$ of $\{1,2, \ldots, n\}, f(T)$ counts the objects of $S$ that satisfy all the properties $P_{j}$ for $j$ in $T$ (and possibly other properties as well). We can think of the unsigned sum $\sum_{T \subseteq\{1,2, \ldots, n\}} f(T)$ as counting ordered pairs $(X, Y)$ where $X$ is a subset of $\{1,2, \ldots, n\}$ and $Y$ is any object satisfying all of the properties $P_{j}$ for $j$ in $X$.

Involution: Given $(X, Y)$, let $x$ be the smallest-indexed property that $Y$ possesses. Then we associate $(X, Y)$ with $(X \oplus x, Y)$.

Exception: The exceptions occur whenever $x$ does not exist, that is, when $Y$ has none of the $n$ properties $P_{1}$ through $P_{n}$. These exceptions come from ordered pairs $(X, Y)$ where $X$ is the empty set. Since the empty set has even size, the summation positively counts the objects in $S$ with no undesirable properties. 


\section{What happens after you D.I.E.?}

Applying the D.I.E method to prove an identity often leads to generalizations that are not immediately obvious. It's important to keep an open mind-not only about the role of particular parameters, but in using the method itself. We show how to prove and then extend in a natural way the identity

$$
\sum_{j=1}^{n} \sum_{k=1}^{n}(-1)^{j+k}\left(\begin{array}{c}
n-1 \\
j-1
\end{array}\right)\left(\begin{array}{c}
n-1 \\
k-1
\end{array}\right)\left(\begin{array}{c}
j+k \\
j
\end{array}\right)=2
$$

mentioned at the beginning of the paper. This will require a modification of our technique, where we analyze repeated involutions (the method of D.I.E.I.E.?) to obtain the extremely attractive solution.

Description: Given positive integers $j, k \leq n,\left(\begin{array}{c}n-1 \\ j-1\end{array}\right)\left(\begin{array}{c}n-1 \\ k-1\end{array}\right)\left(\begin{array}{c}j+k \\ j\end{array}\right)$ counts ordered triples $(X, Y, Z)$ where $X$ is a $j$-subset of $\{1,2, \ldots, n\}$ that must contain $1, Y$ is a $k$-subset of $\{n+1, \ldots, 2 n\}$ that must contain $n+1$, and $Z$ is a subset of $X \cup Y$ with $|Z|=$ $|X|$. The unsigned sum $\sum_{j=1}^{n} \sum_{k=1}^{n}\left(\begin{array}{c}n-1 \\ j-1\end{array}\right)\left(\begin{array}{c}n-1 \\ k-1\end{array}\right)\left(\begin{array}{c}j+k \\ j\end{array}\right)$ counts all such ordered triples $(X, Y, Z)$ as the sizes of $X$ and $Y$ vary between 1 and $n$.

Involution (Part I): Given a triple $(X, Y, Z)$, define $y$ to be the largest number between $n+2$ and $2 n$ such that $y$ does not appear in $Z$. The mapping that sends $(X, Y, Z)$ to $(X, Y \oplus y, Z)$ is a sign-reversing involution, since $j$ is unchanged and $k$ increases or decreases by 1 . Note that $Z$ remains a subset of $X \cup(Y \oplus y)$.

Exception (Part I): The involution fails when $y$ does not exist. This requires $Z$ to contain all the elements $n+2, \ldots, 2 n$. Therefore the exceptions $(X, Y, Z)$ must satisfy $Y=\{n+1, n+2, \ldots, 2 n\}$.

Involution (Part II): Given a triple $(X, Y, Z)$ that has survived as an exception in Part I, define $x$ to be the largest number between 2 and $n$ such that $x$ either appears in both $X$ and $Z$ or is absent from both $X$ and $Z$, that is, $x \in(X \cap Z) \cup\left(X^{c} \cap Z^{c}\right)$. Pair $(X, Y, Z)$ with $(X \oplus x, Y, Z \oplus x)$. Since $Z \subseteq X \cup Y$, it remains true that $Z \oplus x \subseteq(X \oplus x) \cup Y$ and $|Z \oplus x|=|X \oplus x|$.

Exception (Part II): If $|X|<n$, then the involution above is always defined since there is one element $x, 2 \leq x \leq n$, that is missing from $X$ and is therefore missing from $Z$ too. Hence the only exceptions occur when $|X|=n$, forcing $X=\{1,2, \ldots, n\}$ and $|Z|=|X|=n$. Thus the only remaining exceptions are of the form $(X, Y, Z)=$ $(\{1,2, \ldots, n\},\{n+1, n+2, \ldots, 2 n\}, Z)$ where $Z$ contains the elements $n+2, n+$ $3, \ldots, 2 n$, and one other element. That element could be either 1 or $n+1$, and these provide the only two exceptions. Both survivors are weighted positively since $j+k=$ $2 n$ and so is even.

In the preceding analysis, elements 1 and $n+1$ represented the guaranteed members of $X$ and $Y$ respectively. There is no reason to believe we are restricted to specifying only one member of each set. Why not two? three? Why not specify $a$ members of $X$ and $b$ members of $Y$ ? Originally, $X$ and $Y$ were selected from disjoint sets of size $n$. Did they have to be the same size? Why not choose $X$ from an $n$-set and $Y$ from an $m$-set? With very little additional effort, you can modify the above description, involutions, and exceptions to obtain the following generalization:

$$
\sum_{j=a}^{n} \sum_{k=b}^{m}(-1)^{j+k}\left(\begin{array}{c}
n-a \\
j-a
\end{array}\right)\left(\begin{array}{c}
m-b \\
k-b
\end{array}\right)\left(\begin{array}{c}
j+k \\
j
\end{array}\right)=\left(\begin{array}{c}
a+b \\
n-m+b
\end{array}\right) .
$$


Although this identity (and indeed, all of the indentities in this paper) can be proved by algebraic or computational methods (as described in [4] or [8]), we find the D.I.E. approach offers the most satisfying explanation.

\section{Summing up: Why alternating sums are simpler than positive sums}

Although alternating sums may on the surface appear to be harder to explain combinatorially than positive sums, our experience has been the opposite. Given a positive sum with a simple closed form, we have found that the alternating version of that sum usually has a simple (and often simpler) closed form too. For example, $\sum_{k=0}^{n}\left(\begin{array}{l}n \\ k\end{array}\right)=2^{n}$ is simple, but the alternating version has the simpler closed form $\sum_{k=0}^{n}(-1)^{k}\left(\begin{array}{l}n \\ k\end{array}\right)=0$. The nonexistence of a simple closed form for the partial sum $\sum_{k=0}^{m}\left(\begin{array}{l}n \\ k\end{array}\right)$ (as a function of $m$ ) can be established by Gosper's algorithm [8], yet the alternating version of that sum is no trouble at all.

Why should alternating sums be simpler than positive sums? Although we don't have a rigorous answer to that question, the idea shows up in other areas of mathematics. From the analysis of infinite series, we know that if a positive sum converges, then its alternating sum must also converge but the converse is not true. From linear algebra, we know that the permanent of an $n \times n$ matrix is usually hard to calculate (requiring about $n$ ! steps), whereas its alternating sum, the determinant can be computed efficiently (in about $n^{3}$ steps) and it has many nice theoretical properties.

Another explanation why alternating sums are often simpler comes from an identity due to Euler. For any polynomial $p(x)$ of degree less than $n$,

$$
\Delta^{n} p(x)=0,
$$

where $\Delta f(x)=f(x+1)-f(x)$. (Euler's theorem can be proved easily by induction since $\Delta^{n} f(x)=\Delta^{n-1}(\Delta f(x))$ and $\Delta f(x)$ is a polynomial of degree less than $n-1$.) Some of the sums in this paper can be proved using this method.

But from the standpoint of combinatorics, we think that the reason alternating sums are easier than non-alternating sums boils down to this:

\section{Matching is easier than counting.}

Acknowledgment. Special thanks to Doron Zeilberger for his helpful suggestions.

\section{Reference}

1. A. T. Benjamin and J. J. Quinn, Proofs That Really Count: The Art of Combinatorial Proof, MAA, 2003.

2. A. T. Benjamin and J. J. Quinn, Recounting Fibonacci and Lucas Identities, College Math. J. 30 (1999) 359366.

3. F. Franklin, Sur le développement du produit infini $(1-x)\left(1-x^{2}\right)\left(1-x^{3}\right) \cdots$, C. R. Acad. Paris Ser. A 92 (1881) 448-450.

4. R. L. Graham, D. E. Knuth, and O. Patashnik, Concrete Mathematics: A Foundation for Computer Science, Addison-Wesley, 1994.

5. H. Kwong, Always 2, College Math. J. 38 (2007) 154-155. (Solution to Problem 824, proposed by J. Leach, College Math. J. 37 (2006) 144.)

6. I. Pak, On Fine's partition theorems, Dyson, Andrews, and missed opportunities, Math. Intelligencer 25 (2003) 10-16.

7. K. H. Parshall and D. E. Rowe, The Emergence of the American Mathematical Research Community, 19761900: J. J. Sylvester, Felix Klein, and E. H. Moore, AMS and London Mathematical Society, 2002.

8. M. Petkovsek, H. S. Wilf, and D. Zeilberger, $A=B$, A K Peters, 1996.

9. B. Sury, A Parent of Binet's Formula?, Math. Mag. 77 (2004) 308-310. 


\title{
Mathematics in War and Peace
}

\author{
Arthur Neuman, River Falls, Wisconsin
}

In his monumental work War and Peace, first published in the Russian Messenger from 1865 to 1869 , Leo Tolstoy invoked concepts from mathematics and logic in developing his theory of history. Here are some excerpts [1, pp. 821-822]:

The movement of mankind, proceeding from a countless number of human wills, occurs continuously. To comprehend the laws of this movement is the goal of history. But in order to comprehend the laws of continuous movement of the sum of all individual wills, human reason allows for arbitrary, discrete units. The first method of history consists in taking an arbitrary series of continuous events and examining the actions of one person, a king, a commander, as the sum of individual wills, whereas the sum of individual wills is never expressed in the activity of one historical person ... .

Any conclusion of historical science, without the least effort on the part of criticism, falls apart like dust, leaving nothing behind, only as a result of the fact that criticism selects as an object for observation a larger or smaller discrete unit, which it always has a right to do, because any chosen historical unit is always arbitrary.

Only by admitting an infinitesimal unit for observation-a differential of history, that is, the uniform strivings of people - and attaining to the art of integrating them (taking the sums of these infinitesimal quantities) can we hope to comprehend the laws of history.

Tolstoy goes on to use Zeno's paradox to illustrate the dangers inherent in dividing continuous movement into discrete times. Considered a paradox by the ancients, thanks to infinite series in the present-day mathematician's tool box, it is no longer thought to be such.

A well-known so-called sophism of the ancients posits that Achilles can never overtake a tortoise that is walking ahead of him, even though Achilles walks ten times faster than the tortoise: while Achilles covers the distance that separates him from the tortoise, the tortoise will get ahead of him by one tenth of that distance; Achilles covers that one tenth, the tortoise gets ahead by one hundredth, and so on to infinity. The ancients considered this problem insoluble. The nonsensical conclusion (that Achilles will never overtake the tortoise) resulted only from the fact that discrete units of movement were introduced arbitrarily, while the movement of both Achilles and the tortoise was continuous.

Having thus illustrated the futility of a theory of history that considers either an arbitrary series of discrete events or sum of individual wills of a king or commander, Tolstoy mentions the calculus, without naming it.

A new branch of mathematics, having attained to the art of dealing with infinitesimal quantities in other, more complete problems of movement as well, now gives answers to questions that used to seem insoluble.

This new branch of mathematics, unknown to the ancients, in examining questions of movement, allows for infinitesimal quantities, that is, such as restore the main condition of movement (absolute continuity), and thereby corrects the inevitable error that human reason cannot help committing when it examines discrete units of movement instead of continuous movement. The same happens in the search for the laws of historical movement.

\section{Reference}

1. Leo Tolstoy, War and Peace, transl. R. Pevear and L. Volokhonsky, Knopf, 2007. 